\title{
A NOTE ON SOME APPLICATIONS OF SEMI-OPEN SETS
}

\author{
T.M. NOUR
}

Department of Mathematics

University of Jordan

Amman, JORDAN

(Received May 5, 1996 and in revised form August 29, 1996)

\begin{abstract}
The object of the present paper is to study the well known notions of semi-closure, semiinterior, semi-frontier and semi-exterior of a set using the concept of semi-open sets A semi-isolated point of a set is also defined and studied.
\end{abstract}

KEY WORDS AND PHRASES: Semi-closure, semi-interior, semi-isolated point, semi-discrete set, semi-scattered spaces.

1991 AMS SUBJECT CLASSIFICATION CODES: 54A05, 54A20

\section{INTRODUCTION}

A subset $A$ of a topological space $(X, \tau)$ is said to be semi-open [1] if there exists an open set $U$ such that $U \subset A \subset C l(U)$. The complement of a semi-open set is called semi-closed [2] The union of all semi-open sets of $X$ contained in $A$ is called the semi-interior of $A$ [2] and is denoted by $\operatorname{sint}(A)$ The intersection of all semi-closed sets containing $A$ is called the semi-closure of $A$ [2] and is denoted by $s C l(A) \quad s C l(A)-s \operatorname{Int}(A)$ is called the semi-frontier of $A$ [3] and is denoted by $\operatorname{sFr}(A)$ $\operatorname{sInt}(X-A)$ is said to be the semi-exterior of $A[3]$ and is denoted by $s E x t(A)$ In this paper, these notions are further investigated We also introduce and study the concepts of semi-isolated points and semi-scattered spaces

THEOREM 1. For a set $A \subset X$, the following are equivalent

(a) $A$ is dense in $X$

(b) $s C l(A)=X$.

(c) If $B$ is any semi-closed subset of $X$ and $A \subset B$, then $B=X$.

(d) For each $\mathbf{x} \in X$, every semi-open set containing $\mathbf{x}$ has non-empty intersection with $A$

(e) $\operatorname{sint}(X-A)=\emptyset$.

PROOF. (a) $\Rightarrow$ (b). Let $U$ be an open set with $U \subset X-B \subset C l(U)$ Since $U \subset X-A$ and $A$ is dense, therefore $U=\emptyset$ and so $C l(U)=\emptyset$ Hence $B=X$ It follows that the intersection of all semiclosed sets containing $A$ is $X$, that is $s C l(A)=X$.

(b) $\Rightarrow$ (a) Obvious since $s C l(A) \subset C l(A)$ for every $A \subset X$

(b) $\Rightarrow$ (c) and (c) $\Rightarrow$ (d) are obvious.

(d) $\Rightarrow$ (e) If $\operatorname{sInt}(X-A) \neq \emptyset$, then $\operatorname{sInt}(X-A)$ is a non-empty semi-open set However, $(X-A) \cap A=\emptyset$ and since $\operatorname{sint}(X-A) \subset X-A$, we have $\operatorname{sInt}(X-A) \cap A=\emptyset$ This contradicts (d) and means $\operatorname{sint}(X-A)=\emptyset$.

(e) $\Rightarrow$ (b) Since $\operatorname{sInt}(X-A)=X-s C l(A)[1]$, therefore $X=s C l(A)$ 
THEOREM 2. Let $A$ be a subset of the space $X$ Then
(a) $s F r(s \operatorname{Int}(A)) \subset s F r(A)$
(b) $s F r(s C l(A)) \subset s F r(A)$
(c) $s \operatorname{Ext}(X)=\emptyset$
(d) $s \operatorname{Ext}(\emptyset)=X$
(e) $s \operatorname{Ext}(A)=s \operatorname{Ext}[X-s \operatorname{Ext}(A)]$
(f) $\operatorname{sint}(A)=A-s F r(A)$
(g) $\operatorname{sint}(A) \subset s \operatorname{Ext}[s \operatorname{Ext}(A)]$
(h) $X=\operatorname{sint}(A) \cup s E x t(A) \cup s F r(A)$

PROOF. Only the proof of (e) will be given here. We have

$$
\begin{aligned}
s E x t[S-s E x t(A)] & =s E x t[X-s \operatorname{Int}(X-A)] \\
& =s \operatorname{Int}[X-(X-s \operatorname{Int}(X-A))] \\
& =\operatorname{sint}[\operatorname{sint}(X-A)] \\
& =\operatorname{sInt}(X-A)=s \operatorname{Ext}(A) .
\end{aligned}
$$

THEOREM 3. If $A, B \subset X$ such that $s \operatorname{Fr}(A) \cap \operatorname{Fr}(B)=\emptyset$ and $\operatorname{Fr}(A) \cap s \operatorname{Fr}(B)=\emptyset$, then $\operatorname{sInt}(A) \cup \operatorname{sInt}(B)=\operatorname{sInt}(A \cup B)$

PROOF. Let $x \in \operatorname{sInt}(A \cup B)$. Then there exists a semi-open set $U$ such that $x \in U \subset A \cup B$ If $x \in s F r(A)$ then $x \notin F r(B)$, so there exists an open set $V$ containing $x$ with $V \subset B$ or $V \subset X-B$ Assume $V \subset B$. Then $x \in U \cap V \subset B$. Since $U \cap V$ is semi-open, $x \in \operatorname{sint}(B)$ On the other hand, if $V \subset X-B$, then $x \in U \cap V \subset A$ and so $x \in \operatorname{sint}(A)$. If $x \notin s F r(A)$ In particular, suppose that $x \notin s C l(A)$, for otherwise, $x \in \operatorname{sint}(A)$. Then $x \in B \subset s C l(B)$ since $x \in A \cup B$. We may assume that $x \notin s F r(A)$ for otherwise, $x \in \operatorname{sInt}(B) \quad$ Thus $x \notin \operatorname{Fr}(A)$ and the argument now proceeds similarly to the case when $x \notin F r(B)$.

THEOREM 4. A set $A \subset X$ is nowhere dense iff $\operatorname{Int}(s C l(A))=\emptyset$.

PROOF. The proof is obvious since $\operatorname{Int}(C l A)=\operatorname{Int}(s C l(A))$ for every $A \subset X$

DEFINITION 1. Let $A$ be a subset of a topological space $X$. Then

(a) A point $x \in A$ is said to be a semi-isolated point of $A$ if there is a semi-open set $U$ such that $U \cap A=\{x\}$.

(b) A set $A$ is said to be semi-discrete if each point of $A$ is semi-isolated

(c) A space $(X, \tau)$ is said to be semi-scattered if every non-empty subset of $X$ has a semi-isolated point.

It is obvious that every isolated point of $A \subset X$ is semi-isolated. But the converse is not true as can be seen from the following example.

EXAMPLE 1. Consider the usual topology on $\mathbf{R}$. Let $\mathbf{A}=[0,1]$ A subset $U=[1,2)$ of $\mathbf{R}$ is semi-open and $U \cap A=\{1\} .1 \in A$ is a semi-isolated point of $A$ but it is not an isolated point of $A$

REMARK 1. Let $(X, \tau)$ be a topological space and $A \subset X$. Then

(a) A semi-isolated point of $X$ is merely an isolated point. For $\{x\}$ is semi-open iff $\{x\}$ is open. The set of all isolated (semi-isolated) points of a set $A \subset X$ is denoted by $A^{s}\left(A^{s s}\right)$.

(b) A space $X$ is a semi-discrete subset of itself iff $X$ is discrete. Every discrete set is semi-discrete But the converse need not be true as can be seen from the following example.

EXAMPLE 2. The subset $a=[0,1] \times\{0\} \subset R^{2}$ is dense-in-itself but it is semi-discrete. For each $x=(r, 0) \in A$, let $U(x)$ be the open unit disk with nonnegative center coordinates which is tangent to $A$ at the point $x$. Thus $B=U \cap\{x\}$ is semi-open and $\{x\}=B \cap A$. This shows that each point $x \in A$ is a semi-isolated point of $A$. This implies that $A$ is semi-discrete in $R^{2}$. However, $A$ is not discrete since its points are not isolated.

If $A_{s}^{\prime}$ denotes the semi-derived set of $A$, then we have the following theorem 
THEOREM 5. If $A$ is a subset of a space $X$, then
(a) $A_{s}^{\prime} \cap A^{s s}=\emptyset$
(b) $s C l(A)=A_{s}^{\prime} \cap A^{s s}$
(c) $X=A_{s}^{\prime} \cap A^{s s} \cap s \operatorname{Ext}(A)$

PROOF. (a) $x \in A^{s s} \Leftrightarrow$ there is a semi-open set $U$ containing $\mathbf{x}$ such that

$$
\begin{aligned}
& U \cap A=\{x\} \\
\Leftrightarrow & U \cap(A-\{x\})=\emptyset \Leftrightarrow x \notin A_{s}^{\prime} .
\end{aligned}
$$

(b) $x \in s C l(A) \Leftrightarrow U \cap A \neq \emptyset$ for every semi-open set $U$ containing $\mathbf{x}$.

$$
\begin{aligned}
& \Leftrightarrow U \cap(A-\{x\}) \neq \emptyset \text { if } x \notin A \text { or } U \cap(A-\{x\})=\emptyset \text { if } x \in A . \\
& \Leftrightarrow x \in A_{s}^{\prime} \text { or } x \in A^{s s} \Leftrightarrow x \in A_{s}^{\prime} \cup A^{s s} .
\end{aligned}
$$

(c) Obvious in view of parts (a) and (b).

THEOREM 6. If $A \subset X$ is dense, then the following hold:

(a) The semi-isolated points of $A$ are precisely the isolated points of $A$ as a subspace

(b) $A \subset A_{s}^{\prime}$ iff $A^{s}=\emptyset$

PROOF. (a) If $\{x\}=B \cap A$, where $B$ is semi-open, then there is an open set $U$ such that $U \subset B \subset C l(U) . U \cap A \neq \emptyset$ since $A$ is dense in $X . B \neq \emptyset$ implies $U \neq \emptyset$. Thus $U \cap A=\{x\}$ and $\mathbf{x}$ is an isolated point of the subspace $A$. Converse is obvious.

(b) $A^{s}=A^{s s}$ because $A$ is dense in $X$. Since $X=s C l(A)=A_{s}^{\prime} \cup A^{s s}=A_{s}^{\prime} \cup A^{s}$ and $A^{s s} \cap A_{s}^{\prime}=\emptyset$, therefore $A^{s} \cap A_{s}^{\prime}=\emptyset$. Hence $A=A^{s} \cup\left(A \cap A_{s}^{\prime}\right)$. Thus $A \subset A_{s}^{\prime}$ iff $A^{s}=\emptyset$.

THEOREM 7. Every scattered space is semi-scattered.

The following example shows that a semi-scattered space need not be scattered.

EXAMPLE 3. Let $X=\{a, b, c\}$ and $\tau=\{X, \emptyset,\{a\}\}$ be a topology on $X$ Then the set $A=\{b, c\}$ has no isolated points. But every subset of $X$ has semi-isolated points.

ACKNOWLEDGMENT. The author is thankful to the referee for his kind comments and suggestions leading to revision of the paper in the present form.

\section{REFERENCES}

[1] LEVINE, N., Semi-open sets and semi-continuity in topological space, Amer. Math. Monthly 70 (1963), 36-41.

[2] CROSSLEY, S.G. and HILDEBRAND, S.K., Semi-closure, Texas J. Sci. 22 (1971), 99-112

[3] DAS, P., Note on some applications of semi-open sets, Progress Math. 7 (1973), 33-44 


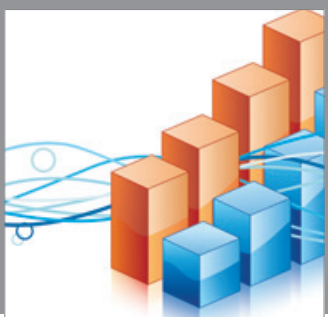

Advances in

Operations Research

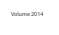

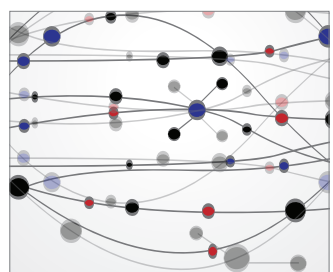

\section{The Scientific} World Journal
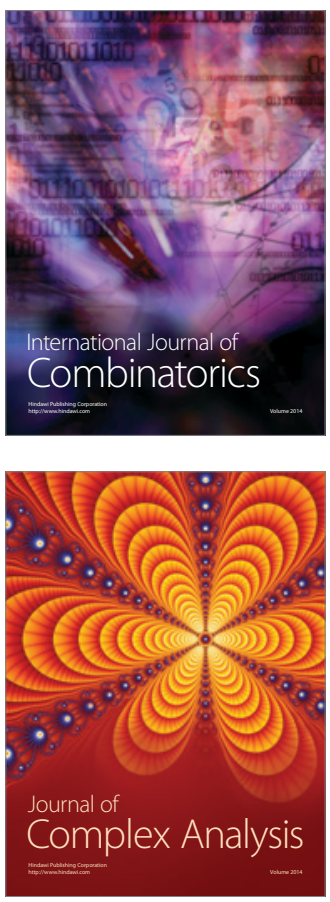

International Journal of

Mathematics and

Mathematical

Sciences
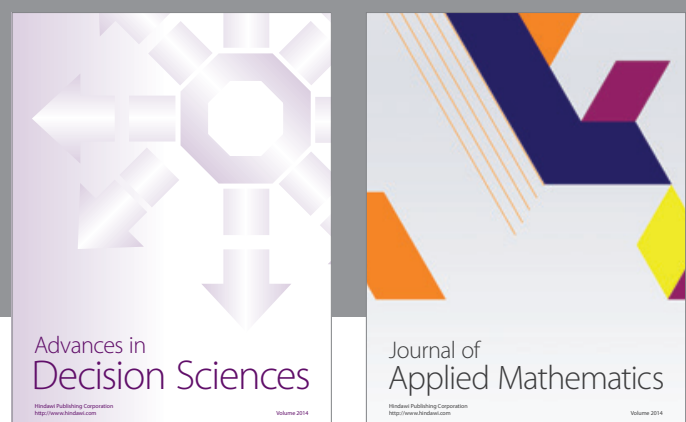

Journal of

Applied Mathematics
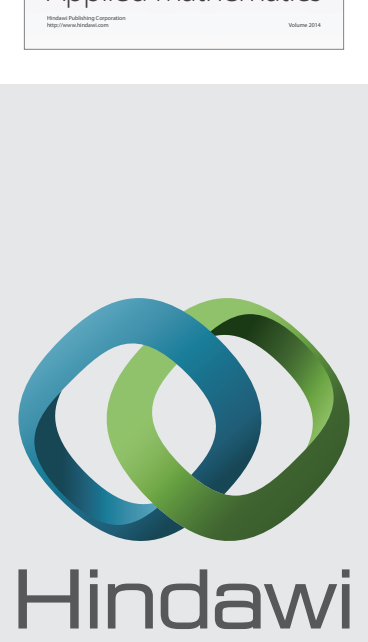

Submit your manuscripts at http://www.hindawi.com
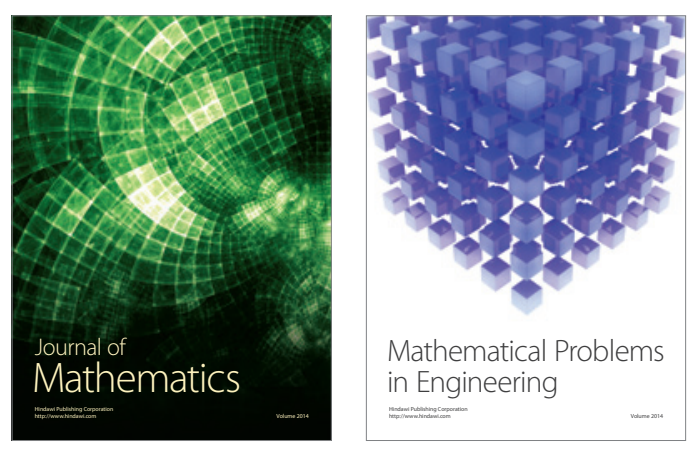

Mathematical Problems in Engineering
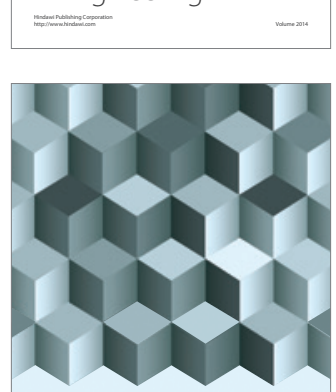

Journal of

Function Spaces
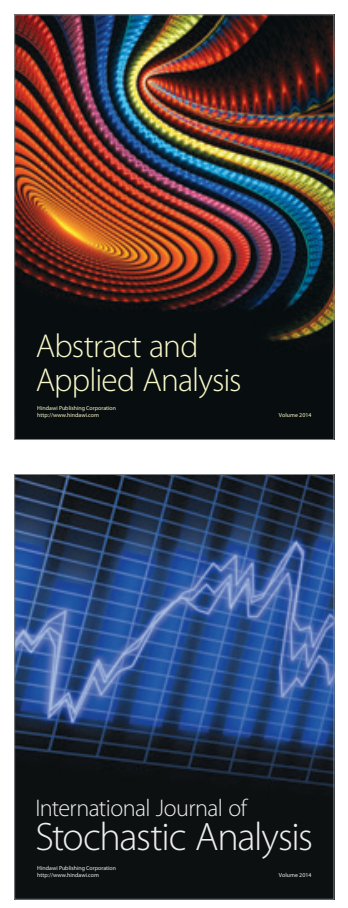

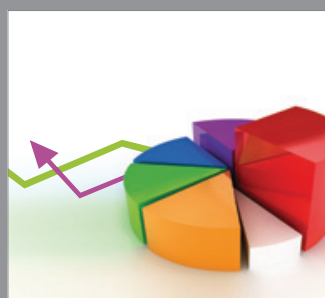

ournal of

Probability and Statistics

Promensencen
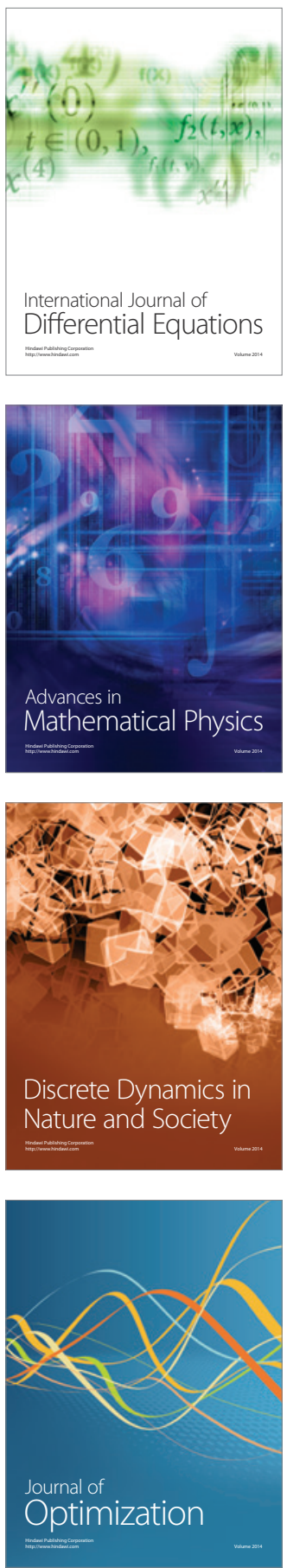